I. Lazzarato ${ }^{1}$, M. Gonzalez-Muñoz ${ }^{2}$, R. Heredia ${ }^{1}$, F. R. Castellar ${ }^{3}$, A. López de la Guía ${ }^{4}$, R. Cabañas ${ }^{1}$, A. Fiandor ${ }^{1}$, J. Dominguez-Ortega ${ }^{1}$

\title{
Successful desensitization procedure to lenalidomide in a patient with delayed hypersensitivity confirmed with a positive LTT
}

${ }^{1}$ Department of Allergy, La Paz Institute for Health Research Hospital (IdiPAZ), Madrid, Spain

${ }^{2}$ Department of Immunology, La Paz Hospital, Madrid, Spain

${ }^{3}$ Pharmacy Service, La Paz Hospital, Madrid, Spain

${ }^{4}$ Department of Hematology, La Paz Hospital, Madrid, Spain

\section{KEY WORDS \\ Drug allergy; desensitization; lenalidomide; multiple myeloma; lymphocyte transformation test.}

\author{
Corresponding author \\ Ilaria Lazzarato \\ Department of Allergy \\ Hospital Universitario La Paz \\ Paseo de la Castellana 261 \\ 28046 Madrid, Spain \\ E-mail: ilarialaz@yahoo.it
}

\section{DOI}

10.23822/EurAnnACI.1764-1489.134

To the Editor,

Lenalidomide is an immunomodulatory oral synthetic-derivative of thalidomide which is indicated in association with dexamethasone in refractory multiple myeloma (MM) and when it relapses. Lenalidomide acts inducing apoptosis of tumour cells and changes in micro-environmental conditions of tumour stroma and angiogenesis and stimulating the host immune response through the activation of cytotoxic T-lymphocytes and Natural Killer-cells $(1,2)$.

Adverse drug reactions (ADRs) to lenalidomide range from 6\% to $43 \%$, mostly morbilliform, urticarial and maculopapular exanthema, occurring within the first month of treatment (3). Some cases of severe cutaneous ADRs have also been reported such as Drug Reaction with Eosinophilia and Systemic Symptoms (DRESS), erythema multiforme, Stevens-Johnson syndrome (SJS) and toxic epidermal necrolysis (TEN). According to a meta-analysis conducted on ten trials, the overall incidence of all-grade and high-grade rash was $27.2 \%$ and $3.6 \%$, respectively (4). In most cases, the relationship between the drug and rash development was suggestive leading to the removal of the drug, but they could not be ascertained with an objective test. We present the case of a 77-year-old man with MM (lambdalight-chain disease, stage Durie Salmon IIIB, ISS3) diagnosed 2 years before, with renal impairment and bone lesions. His personal background included a colostomy for diverticulitis, inguinal herniorraphy and a transurethral resection for prostatic hypertrophy. He presented a previous allergic reaction to colistin, 
but he had no history of either food or latex allergy, rhinitis or asthma. A third-line treatment with lenalidomide $(10 \mathrm{mg} / 24 \mathrm{~h}$ on days 1 to 21 of a 28-day cycle)-dexamethasone was initiated in February 2017 upon evidence of disease progression. In December 2017, after 5 days on the $10^{\text {th }}$ cycle, he experienced an acute pruritic exanthema, developing a generalized morbilliform eruption on the trunk and folds with residual flaking skin. No mucosa involvement was observed. Neither pustules, vesicles or blisters were present. No eosinophilia, enlarged lymph nodes, elevated creatinine or hepatitis signs were detected. With the suspicion of a toxicodermic reaction, the discontinuation of lenalidomide was decided and the patient was treated with oral prednisolone for two weeks.

In an attempt to clarify the underlying mechanism of this reaction, a lymphocyte transformation test (LTT) with lenalidomide was performed. This method is performed by incubating fresh peripheral-blood mononuclear cells from patient previously separated over a density gradient (Histopaque-1077, Sigma-Aldrich) for 6 days at $10^{6}$ cells $/ \mathrm{mL}$, at different concentration of the suspected drug. In this case, the test was performed in triplicates with lenalidomide at $0.1 \mu \mathrm{g} / \mathrm{mL}-100 \mu \mathrm{g} / \mathrm{mL}$. Drug was provided by the Hospital Pharmacy. Phytohemagglutinin $(5 \mu \mathrm{g} / \mathrm{mL})$ was used as positive control. Proliferation was determined by the addition of $\left({ }^{3} \mathrm{H}\right)$ thymidine $(0.5 \mu \mathrm{Ci} /$ well $)$ for the final 18 hours of the incubation period. The result is expressed as stimulation index (SI), which is the relationship between proliferation of lymphocytes in the presence or absence of the drug (basal proliferation). A positive result is suggestive of sensitization to the drug although a negative result does not exclude sensitization (5). The positive control result was 181 counts per minute (cpm) and that of basal proliferation was 58 $\mathrm{cpm}$. A positive response, defined as an SI of over 2 in at least

Figure 1 - Lymphocyte transformation test results for lenalidomide. The test is considered positive when the stimulation index (SI) is greater than 2. Stripped bars represent SIs of three healthy controls. Open bars show SIs of the patient.

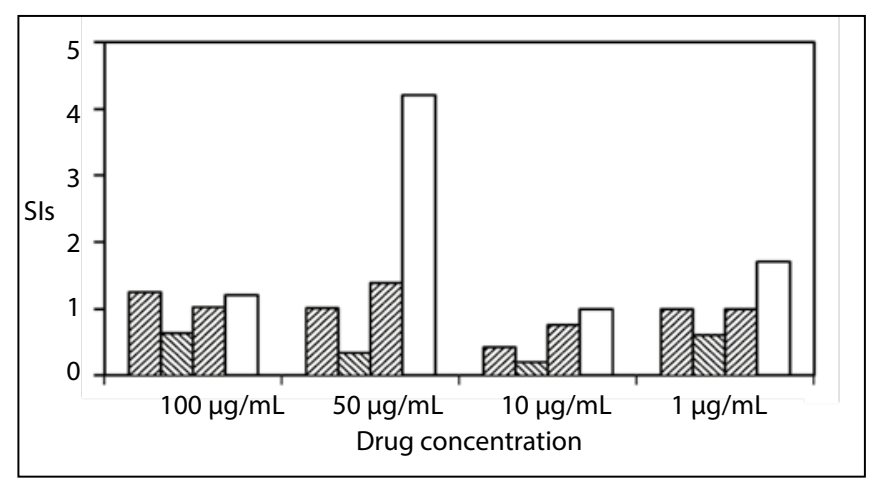

one of the doses tested, was obtained with lenalidomide. LTT with lenalidomide in 3 different healthy controls showed no proliferative responses (figure 1).

A progression of the disease was verified in May 2018 and the haematologist decided to reintroduce the drug as the treatment of choice. Different strategies have been suggested for hypersensitivity dermatologic reactions induced by lenalidomide, including drug discontinuation or antihistamine and corticosteroid premedication. There are some few published reports of rapid inpatient desensitization in patients with acute urticarial rash (6) and an outpatient 6-week desensitization protocol for a target dose of $10 \mathrm{mg}$, in 5 patients with cutaneous delayed reaction (7). Considering the presence of an immunological mechanism causing the reaction and assessing all possible treatment options, we decided to perform a desensitization procedure. After assessing the safety of drug handling for small doses, a first attempt was initiated with a dose escalation procedure, rising daily the dose from $1 \mathrm{mg}$, which was planned to last 5 days $(1,2,2.5,5$, $10 \mathrm{mg}$ ). Under specialist supervision in our outpatient clinic, the heart rate, blood pressure, pulse oximetry and peak-flow rate were monitored. This first attempt was interrupted at the third day of treatment, four hours later to the drug intake, the patient reported the presence of an intense armpits and scalp itching in absence of skin lesions, which persisted 48 hours after the removal of the drug. Cutaneous symptoms were accompanied by a single and self-limited episode of diarrhoea. Since the first attempted desensitization protocol failed, we designed a new one with dose escalation every 3 days based on previous recommendations in delayed reactions to allopurinol (8). We also restarted the procedure from a lower initial dose, adding concomitant bilastine $20 \mathrm{mg} / 24 \mathrm{~h}$. Table I shows the adjusted 14-day protocol that was carried out from the initial dose of $0.1 \mathrm{mg}$ of lenalidomide up to $10 \mathrm{mg} / 24 \mathrm{~h}$ according to the dose prescribed by the hematologist. Escalating doses were tolerated, achieving the dose of $10 \mathrm{mg}$, since he continued to receive this daily dose of $10 \mathrm{mg}$ for the next two months, without appearance of new episodes of itching, diarrhea, or skin involvement.

We report the case of a patient who developed a delayed erythematous morbilliform skin eruption in course of taking lenalidomide. For the first time, the implication of this drug was established by a positive LTT. Although LTT has not been completely standardized yet for many drugs, it should be considered a useful in vitro diagnostic tool, especially in non-immediate reactions. LTT reflects the reactivation and proliferation of memory cells that are present in the peripheral blood of allergic patients and it is not necessarily associated with more severe clinical symptoms and a dose-response pattern (9). For some drugs, LTT could offer a better diagnostic value than patch and intradermal tests to identify allergic subjects $(10,11)$. For drugs such as beta-lactams, LTT can reach a $92.8 \%$ of specificity, obtaining positive results even 10 or more years after the occur- 
rence of the reaction, without further exposure to the drug (12). Moreover, LTT is safe for patients, which is absolutely relevant for severe reactions. In addition, we could propose an effective and safe alternative with a 14-day desensitization procedure, although it needs to be further validated in more patients.

In summary, this is the first reported case of a patient with hypersensitivity to lenalidomide, demonstrated by a positive LTT, in whom a short successful outpatient oral desensitization procedure was performed.

\section{References}

1. Rajkumar SV. Multiple myeloma: 2016 update on diagnosis, risk-stratification, and management. Am J Hematol 2016; 91:719-34.

2. Anderson KC. Lenalidomide and thalidomide: Mechanisms of action-similarities and differences. Semin Hematol 200; 42:S3-S8.

3. Patrizi A, Venturi M, Dika M, Maibach H, Tacchetti P, Brandi G. Cutaneous adverse reactions linked to targeted anticancer therapies bortezomib and lenalidomide for multiple myeloma: new drugs, old side effects. Cutan Ocul Toxicol 2014;33:1-6

4. Nardone B, Wu S, Garden BC, West DP, Reich LM, Lacouture ME. Risk of rash associated with lenalidomide in cancer patients: a systematic review of the literature and meta-analysis. Clin Lymphoma Myeloma Leuk 2013;13:424-9.
5. Pichler WJ, Tilch J. The lymphocyte transformation test in the diagnosis of drug hypersensitivity. Allergy 2004: 59: 809-20.

6. Seki JT, Banglawala S, Lentz EM, Reece DE. Desensitization to lenalidomide in a patient with relapsed multiple myeloma. Clin Lymphoma Myeloma Leuk 2013; 13:162-5

7. Lee MJ, Wickner P, Fanning L, Schlossman R, Richardson P, Laubach J, et al. Lenalidomide desensitization for delayed hypersensitivity reactions in 5 patients with multiple myeloma. Br J Haematol. 2014; 167:127-31

8. Fam AG, Dunne SM, Iazzetta J, Paton TW. Efficacy and safety of desensitization to allopurinol following cutaneous reactions. Arthritis Rheum 2001; 44:231-8.

9. Lochmatter P and Pichler WJ. In vitro tests in drug hypersensitivity diagnosis. Immunol Allergy Clin North Am 2009; 29: 537-54.

10. Gonzalez-Cavero L, Dominguez-Ortega J, Gonzalez-Muñoz M, Mayor-Ibarguren A, Tomás M, Fiandor A, et al. delayed allergic reaction to terbinafine with a positive lymphocyte transformation test. J Investig Allergol Clin Immunol 2017; 27: 136-7.

11. Monge-Ortega OP, Cabañas R, Fiandor A, Domínguez-Ortega J, González-Muñoz M, Quirce S, et al. Overlap between DRESS syndrome and exanthema induced by sulfadiazine in a patient treated with sulfamethoxazole: utility of the lymphocyte transformation test for identification of the culprit drug. J Investig Allergol Clin Immunol 2018; 28:132-4.

12. Luque I, Leyva L, Torres MJ, Rosal M, Mayorga C, Segura JM, Blanca M, Juárez C. In vitro T-cell responses to beta-lactam drugs in immediate and nonimmediate allergic reactions. Allergy 2001; 56:611-8. 
1. DENOMINAZIONE DEL MEDICINALE. AYRINAL $20 \mathrm{mg}$ compresse. 2. COMPOSIZIONE QUALITATIVA E QUANTITATIVA. Ogni compressa contiene $20 \mathrm{mg}$ di bilastina. Per l'elenco completo degli eccipienti, vedere paragrafo 6.1. 3. FORMA FARMACEUTICA. Compressa. Compresse bianche, ovali, biconvesse con linea di incisione (lunghezza $10 \mathrm{~mm}$, larghezza $5 \mathrm{~mm}$ ). La linea di incisione sulla compressa serve solo per agevolarne la rottura al fine di ingerire la compressa più facilmente e non per dividerla in dosi uguali. 4. INFORMAZIONI CLINICHE. 4.1. Indicazioni terapeutiche. Trattamento sintomatico della rinocongiuntivite allergica (stagionale e perenne) e dell'orticaria. AYRINAL è indicato negli adulti e negli adolescenti (12 anni di età ed oltre). 4.2. Posologia e modo di somministrazione. Posologia. Adulti e adolescenti (12 anni di età ed oltre). $20 \mathrm{mg}$ di bilastina (1 compressa) una volta al giorno per alleviare i sintomi della rinocongiuntivite allergica (SAR e PAR) e dell'orticaria. La compressa deve essere assunta un'ora prima o due ore dopo l'assunzione di cibo o succhi di frutta (vedere paragrafo 4.5). Durata del trattamento: Per la rinocongiuntivite allergica il trattamento deve essere limitato al periodo di esposizione agli allergeni. Per la rinite allergica stagionale il trattamento può essere interrotto dopo la scomparsa dei sintomi e ripreso alla loro ricomparsa. Nella rinite allergica perenne può essere proposto ai pazienti un trattamento continuato durante il periodo di esposizione agli allergeni. Nell'orticaria la durata del trattamento dipende dal tipo, dalla durata e dal decorso dei disturbi. Popolazioni speciali: Anziani: Non sono necessari aggiustamenti del dosaggio nei pazienti anziani (vedere paragrafi 5.1 e 5.2 ). Compromissione renale: Studi condotti negli adulti in speciali gruppi di rischio (pazienti con compromissione renale) indicano che non è necessario un aggiustamento della dose negli adulti (vedere paragrafo 5.2). Compromissione epatica: Non esiste esperienza clinica in pazienti adulti con compromissione epatica. Tuttavia, dato che la bilastina non viene metabolizzata e viene eliminata immodificata nell'urina e nelle feci, non si prevede che la compromissione epatica aumenti l'esposizione sistemica oltre il margine di sicurezza nei pazienti adulti. Pertanto, non è necessario alcun aggiustamento del dosaggio nei pazienti adulti con compromissione epatica (vedere paragrafo 5.2). Popolazione pediatrica: - Bambini dai 6 agli 11 anni di età con un peso corporeo di almeno $20 \mathrm{~kg}$. Bilastina $10 \mathrm{mg}$ compresse orodispersibili e bilastina $2,5 \mathrm{mg} / \mathrm{ml} \mathrm{soluzione} \mathrm{orale} \mathrm{sono}$ appropriate per la somministrazione a questa popolazione. - Bambini sotto i 6 anni di età e sotto i $20 \mathrm{~kg}$. I dati attualmente disponibili sono descritti nei paragrafi 4.4, 4.8, 5.1 e 5.2, ma non possono essere effettuate raccomandazioni relativamente alla posologia. Pertanto la bilastina non deve essere usata in questa fascia di età. La sicurezza e l'efficacia di bilastina nei bambini con compromissione renale ed epatica non sono state stabilite. Modo di somministrazione: Uso orale. La compressa deve essere deglutita con acqua. Si raccomanda di assumere la dose giornaliera in un'unica somministrazione. 4.3. Controindicazioni. Ipersensibilità al principio attivo o ad uno qualsiasi degli eccipienti elencati al paragrafo 6.1. 4.4. Avvertenze speciali e precauzioni d'impiego. Popolazione pediatrica: L'efficacia e la sicurezza della bilastina nei bambini al di sotto dei 2 anni di età non sono state stabilite ed esiste una limitata esperienza clinica nei bambini tra i 2 e i 5 anni di età, pertanto la bilastina non deve essere usata in queste fasce di età. Nei pazienti con compromissione renale da moderata a grave la co-somministrazione della bilastina con inibitori della P-glicoproteina, quali ad esempio chetoconazolo, eritromicina, ciclosporina, ritonavir o diltiazem, può aumentare i livelli plasmatici della bilastina e pertanto aumentare il rischio di effetti avversi. Pertanto, la co-somministrazione della bilastina ed inibitori della P-glicoproteina deve essere evitata in pazienti con compromissione renale da moderata a grave. 4.5. Interazioni con altri medicinali ed altre forme di interazione. Studi di interazione sono

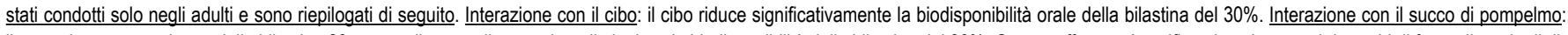
l'assunzione concomitante della bilastina $20 \mathrm{mg}$ con il succo di pompelmo diminuisce la biodisponibilità della bilastina del $30 \%$. Questo effetto può verificarsi anche con altri succhi di frutta. II grado di diminuzione della biodisponibilità può variare a seconda dei diversi produttori e dei frutti. II meccanismo di questa interazione è l'inibizione dell'OATP1A2, un trasportatore di uptake per il quale la bilastina è un substrato (vedere paragrafo 5.2). I medicinali che sono substrati o inibitori dell'OATP1A2, come ritonavir o rifampicina, possono analogamente avere il potenziale di diminuire la concentrazione plasmatica della bilastina. Interazione con chetoconazolo o eritromicina: l'assunzione concomitante di bilastina $20 \mathrm{mg}$ una volta al giorno e chetoconazolo $400 \mathrm{mg}$ una volta al giorno 0 eritromicina $500 \mathrm{mg}$ tre volte al giorno ha aumentato l'AUC della bilastina di 2 volte e la $C_{\max }$ di 2-3 volte. Questi cambiamenti possono essere spiegati dall'interazione con le proteine di trasporto intestinale, in quanto la bilastina è un substrato per P-gp e non viene metabolizzata (vedere paragrafo 5.2). Questi cambiamenti non sembrano avere effetti sul profilo di sicurezza della bilastina e chetoconazolo 0 eritromicina, rispettivamente. Analogamente altri medicinali che sono substrati o inibitori di P-gp, come la ciclosporina, possono potenzialmente aumentare la concentrazione plasmatica della bilastina. Interazione con diltiazem: l'assunzione concomitante della bilastina $20 \mathrm{mg}$ una volta al giorno e diltiazem $60 \mathrm{mg}$ una volta al giorno ha aumentato la $\mathrm{C}_{\max }$ della bilastina del $50 \%$. Questo effetto può essere spiegato dall'interazione con le proteine di trasporto intestinale (vedere paragrafo 5.2) e non sembra avere effetti sul profilo di sicurezza della bilastina. Interazione con alcool: la performance psicomotoria dopo l'assunzione concomitante di alcool e della bilastina $20 \mathrm{mg}$ una volta al giorno è stata simile a quella osservata dopo l'assunzione di alcool e placebo. Interazione con lorazepam: l'assunzione concomitante della bilastina $20 \mathrm{mg}$ una volta al giorno e lorazepam $3 \mathrm{mg}$ una volta al giorno per 8 giorni non ha potenziato gli effetti sedativi sul SNC del lorazepam. Popolazione pediatrica: Sono stati effettuati studi di interazione solo negli adulti. Poiché non c'è esperienza clinica riguardo l'interazione della bilastina con altri medicinali, cibo o succhi di frutta nei bambini, i risultati ottenuti negli studi di interazione nella popolazione adulta devono essere presi in considerazione quando la bilastina viene prescritta ai bambini. Non esistono dati clinici nei bambini per dimostrare se cambiamenti nell'AUC $0 \mathrm{C}_{\max }$ dovuti ad interazioni influenzano il profilo di sicurezza della bilastina. 4.6. Fertilità, gravidanza e allattamento. Gravidanza: i dati relativi all'uso della bilastina in donne in gravidanza non esistono o sono in numero limitato. Studi condotti sugli animali non indicano la presenza di effetti negativi diretti o indiretti riguardanti la tossicità riproduttiva, il parto o lo sviluppo postnatale (vedere paragrafo 5.3 ). A scopo precauzionale, è preferibile evitare l'uso di AYRINAL durante la gravidanza. Allattamento: L'escrezione della bilastina nel latte non è stata studiata nell'uomo. I dati farmacocinetici disponibili sugli animali hanno evidenziato escrezione della bilastina nel latte (vedere paragrafo 5.3). La decisione in merito alla continuazione 0 allinterruzione dell'allattamento 0 ad interrompere/astenersi dalla terapia con AYRINAL deve tenere in considerazione il beneficio dell'allattamento per il bambino e il beneficio della terapia con la bilastina per la madre. Fertilità: non esistono dati clinici oppure sono in numero limitato. Uno studio condotto nei ratti non ha indicato alcun effetto negativo sulla fertilità (vedere paragrafo 5.3). 4.7. Effetti sulla capacità di guidare veicoli e sull'uso di macchinari. Uno studio condotto negli adulti per stabilire gli effetti della bilastina sulla capacità di guidare ha dimostrato che il trattamento con dosi di $20 \mathrm{mg}$ non influenza la capacità di guida. Tuttavia, dato che la risposta individuale al medicinale può essere differente, i pazienti devono essere avvertiti di non guidare o usare macchinari fino a quando non avranno stabilito la propria risposta alla bilastina. 4.8. Effetti indesiderati. Sintesi del profilo di sicurezza in pazienti adulti e adolescenti: L'incidenza di eventi avversi in pazienti adulti e adolescenti affetti da rinocongiuntivite allergica o da orticaria idiopatica cronica trattati con 20 mg di bilastina nei trial clinici è stato paragonabile all'incidenza in pazienti trattati con placebo (12,7\% rispetto a $12,8 \%)$. Durante lo sviluppo clinico, sono stati condotti studi di fase II e III che hanno incluso 2525 pazienti adulti ed adolescenti trattati con diversi dosaggi di bilastina, di cui 1697 sono stati trattati con bilastina $20 \mathrm{mg}$. In questi studi 1362 pazienti hanno ricevuto placebo. Le reazioni avverse più comunemente segnalate dai pazienti che hanno ricevuto $20 \mathrm{mg}$ di bilastina per lindicazione rinocongiuntivite allergica o orticaria idiopatica cronica sono state mal di testa, sonnolenza, capogiri e affaticamento. Questi eventi avversi si sono verificati con una frequenza paragonabile nei pazienti trattati con placebo. Tabella riassuntiva delle reazioni avverse in pazienti adulti e adolescenti: Nella tabella che segue sono riportate le reazioni avverse possibilmente correlate alla bilastina e segnalate in oltre lo $0,1 \%$ dei pazienti trattati con $20 \mathrm{mg}$ di bilastina nel corso dello sviluppo clinico ( $\mathrm{N}=1697$ ). Le frequenze sono assegnate come segue: Molto comune ( $\geq 1 / 10$ ); Comune (da $\geq 1 / 100 \mathrm{a}<1 / 10$ ); Non comune (da $\geq 1 / 1.000 \mathrm{a}<1 / 100$ ); Raro (da $\geq 1 / 10.000 \mathrm{a}<1 / 1.000$ ); Molto raro (<1/10.000); Non nota (la frequenza non può essere definita sulla base dei dati disponibili). Le reazioni rare, molto rare e con frequenza non nota non sono state incluse nella tabella.

\begin{tabular}{|c|c|c|c|c|}
\hline \multicolumn{2}{|r|}{ Classificazione per Sistemi ed Organi } & \multirow{2}{*}{$\begin{array}{c}\text { Bilastina } \\
20 \mathrm{mg} \\
\mathrm{N}=1697\end{array}$} & \multirow{2}{*}{$\begin{array}{l}\text { Bilastina } \\
\text { Tutte le dosi } \\
\mathrm{N}=2525\end{array}$} & \multirow{2}{*}{$\begin{array}{l}\text { Placebo } \\
\mathrm{N}=1362\end{array}$} \\
\hline Frequenza & Reazione avversa & & & \\
\hline \multicolumn{5}{|c|}{ Infezioni e infestazioni } \\
\hline Non comune & Herpes orale & $2(0,12 \%)$ & $2(0,08 \%)$ & $0(0.0 \%)$ \\
\hline Non comune & Aumento dell'appetito & $10(0,59 \%)$ & $11(0,44 \%)$ & $7(0.51 \%)$ \\
\hline \multicolumn{5}{|l|}{ Disturbi psichiatrici } \\
\hline & Ansia & $6(0,35 \%)$ & $8(0,32 \%)$ & $0(0.0 \%)$ \\
\hline Non comune & Insonnia & $2(0,12 \%)$ & $4(0,16 \%)$ & $0(0.0 \%)$ \\
\hline Comune & Cefalea & $68(4.01 \%)$ & $90(3.56 \%)$ & $46(3.38 \%)$ \\
\hline Non comune & Capogiri & $14(0.83 \%)$ & $23(0.91 \%)$ & $8(0.59 \%)$ \\
\hline \multicolumn{5}{|c|}{ Disturbi dell'orecchio e del labirinto } \\
\hline \multirow{2}{*}{ Non comune } & Tinnito & $2(0,12 \%)$ & $2(0,08 \%)$ & $0(0.0 \%)$ \\
\hline & Vertigini & $3(0,18 \%)$ & $3(0,12 \%)$ & $0(0.0 \%)$ \\
\hline
\end{tabular}

